\title{
Deep eutectic solvent as a highly efficient reaction media for the one-pot synthesis of benzo-fused seven-membered heterocycles
}

\author{
Ahmad Shaabani*, Seyyed Emad Hooshmand, Mohammad Taghi Nazeri, Ronak Afshari and Shima \\ Ghasemi
}

Faculty of Chemistry, Shahid Beheshti University, G. C., P. O. Box 19396-4716, Tehran, Iran

Corresponding author: a-shaabani@sbu.ac.ir; Tel.: +98-212-990-2800

\section{ARTICLE INFO}

\section{ABSTRACT}

\section{Article history:}

Received

Received in revised form

Accepted

Available online

Keywords:

Deep Eutectic Solvent

Benzodiazepines

Benzoxazepines

Multicomponent reactions

Green chemistry

\section{Introduction}

Deep Eutectic Solvents (DESs) as a solvent/organocatalyst have gained widespread attention in the last decade. DESs are simple and inexpensive to prepare, generate no waste, require no purification, and possess high atom economy aspects thus embrace the principles of Green Chemistry. ${ }^{1}$ DESs are prepared by mixing high melting-point precursors which in liquid form possess strong hydrogen-bonding interactions that reduce the ability of the components to crystallize., 3 The most studied series of DESs are those prepared using choline chloride as a donor compound with components including urea, sugars, carboxylic acids and ethylene glycol or $\mathrm{M}^{2+}$ ions such as $\mathrm{Sn}^{2+2}$. One of the most notable properties of DESs is their high viscosity. This may result from many factors including the presence of an extensive hydrogen-bonding network, relatively large ion sizes and electrostatic forces within the liquid. Furthermore, they possess a very low volatility, a wider liquid temperature range than molecular solvents and are generally inflammable. DESs can be used to create alloys, ${ }^{4}$ and metal nanoparticles. ${ }^{1,5}$ In addition, these systems have been investigated for utilization in pharmaceutical applications, ${ }^{6}$ extraction, ${ }^{7}$ surfactant chemistry ${ }^{8}$ and various organic reactions. ${ }^{9,10}$

Multicomponent coupling reactions (MCCRs) are highly efficient strategies to achieve the rapid assembly of complex products, especially carbon-carbon and carbon-heteroatom bond-forming reactions in the synthesis of heterocycles ${ }^{11-13}$ and natural products. ${ }^{14}$ Seven-membered heterocyclic systems hold a prominent position as they constitute an integral part of natural alkaloids, antibiotics and synthetic drugs. ${ }^{15}$ Benzo-fused sevenmembered heterocycles containing two heteroatoms at positions
1 and 4, such as 1,4-benzodiazepine and 1,4-benzoxazepine derivatives, are used in medicinal chemistry due to their wide spectrum of biological activities (Fig. 1). ${ }^{16-21}$ Biologically active 1,4-benzodiazepines have demonstrated considerable utility in central nervous system (CNS)-drug design, and as key intermediates for the preparation of fused ring compounds. ${ }^{22}$ Some examples are diazepam and chlorodiazepoxide that act as anti-anxiety drugs. Amongst the examples of 1,4-benzoxazepines of medicinal interest are a non-nucleoside HIV-1 reverse transcriptase inhibitor, ${ }^{23}$ a histamine receptor agonist ${ }^{19}$ and calcium antagonists, ${ }^{24}$ as well as antidepressants and analgesics. ${ }^{25}$

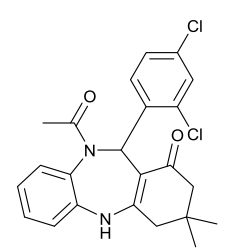

HCV NS5B inhibitor

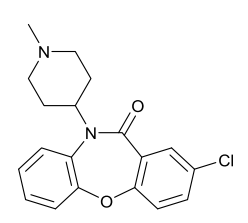

loxapine
Figure 1. Representative examples of biologically active compounds containing the benzo-fused seven-membered heterocycle scaffold

\section{Results and Discussion}

As part of our continued interest in developing new methods for the synthesis of heterocyclic compounds ${ }^{26-30}$ using green chemistry, ${ }^{31,32}$ ionic liquids ${ }^{33}$ and DESs,${ }^{34-36}$ herein, we describe a highly efficient protocol for the synthesis of tricyclic 1,4benzodiazepines and 1,4-benzoxazepines 4 via a one-pot, threecomponent reaction in a choline chloride and urea based DES at $80^{\circ} \mathrm{C}$ without using metal or acid catalysts nor organic solvents. This protocol involves the reaction of an $o$-phenylenediamine or 
2-aminophenol 1, dimedone 2 , and various aromatic aldehydes 3 (Scheme 1).

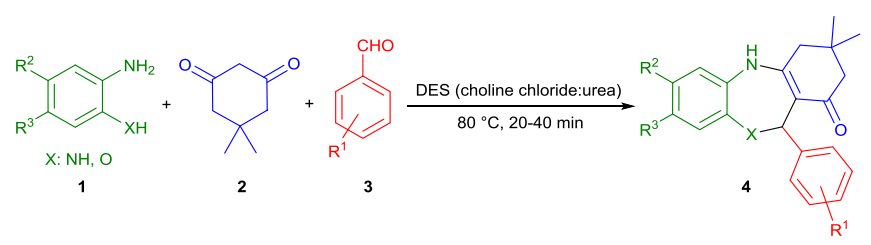

Scheme 1. Synthesis of benzo-fused seven-membered heterocycles via a three-component reaction in DES

The scope and limitations of this reaction were explored by utilizing various $o$-phenylenediamines or 2-aminophenols (1), dimedone (2) and various benzaldehyde derivatives (3) (Table 1). As indicated in Table 1, the benzodiazepines were synthesized in higher yields (80-94\%) with shorter reaction times (20-30 min) relative to the benzoxazepines $(68-88 \%, 30-40 \mathrm{~min})$.

Table 1. Synthesis of tricyclic 1,4-benzodiazepines and 1,4benzoxazepines

\begin{tabular}{|c|c|c|c|c|c|c|}
\hline Entry & $\mathrm{R}^{1}$ & $\mathrm{R}^{2}$ & $\mathrm{R}^{3}$ & $X$ & Product & Yield $^{\mathrm{a}}$ \\
\hline 1 & $4-\mathrm{NO}_{2}$ & $\mathrm{H}$ & $\mathrm{H}$ & $\mathrm{NH}$ & $4 a$ & $94 \%$ \\
\hline 2 & $4-\mathrm{Cl}$ & $\mathrm{H}$ & $\mathrm{H}$ & $\mathrm{NH}$ & $4 b$ & $92 \%$ \\
\hline 3 & 4-Me & $\mathrm{H}$ & $\mathrm{H}$ & $\mathrm{NH}$ & $4 c$ & $82 \%$ \\
\hline 4 & 4-MeO & $\mathrm{H}$ & $\mathrm{H}$ & $\mathrm{NH}$ & $4 d$ & $85 \%$ \\
\hline 5 & $3-\mathrm{NO}_{2}$ & $\mathrm{H}$ & $\mathrm{H}$ & $\mathrm{NH}$ & $4 e$ & $92 \%$ \\
\hline 6 & $2-\mathrm{NO}_{2}$ & $\mathrm{H}$ & $\mathrm{H}$ & $\mathrm{NH}$ & $4 f$ & $80 \%$ \\
\hline 7 & $2,4-\mathrm{Cl}$ & $\mathrm{H}$ & $\mathrm{H}$ & $\mathrm{NH}$ & $4 g$ & $88 \%$ \\
\hline 8 & $3-\mathrm{NO}_{2}$ & $\mathrm{Me}$ & $\mathrm{Me}$ & $\mathrm{NH}$ & $4 h$ & $86 \%$ \\
\hline 9 & $\mathrm{H}$ & $\mathrm{H}$ & $\mathrm{H}$ & $\mathrm{O}$ & $4 i$ & $80 \%$ \\
\hline 10 & $4-\mathrm{Cl}$ & $\mathrm{H}$ & $\mathrm{H}$ & $\mathrm{O}$ & $4 \mathbf{j}$ & $86 \%$ \\
\hline 11 & $4-\mathrm{Br}$ & $\mathrm{H}$ & $\mathrm{H}$ & $\mathrm{O}$ & $4 k$ & $83 \%$ \\
\hline 12 & 4-Me & $\mathrm{H}$ & $\mathrm{H}$ & $\mathrm{O}$ & 41 & $75 \%$ \\
\hline 13 & $4-\mathrm{NO}_{2}$ & $\mathrm{H}$ & $\mathrm{H}$ & $\mathrm{O}$ & $4 m$ & $88 \%$ \\
\hline 14 & 4-Ph & $\mathrm{H}$ & $\mathrm{H}$ & $\mathrm{O}$ & $4 n$ & $74 \%$ \\
\hline 15 & $3-\mathrm{NO}_{2}$ & $\mathrm{H}$ & $\mathrm{H}$ & $\mathrm{O}$ & 40 & $85 \%$ \\
\hline 16 & $3-\mathrm{OH}$ & $\mathrm{H}$ & $\mathrm{H}$ & $\mathrm{O}$ & $4 p$ & $68 \%$ \\
\hline 17 & $2-\mathrm{Cl}$ & $\mathrm{H}$ & $\mathrm{H}$ & $\mathrm{O}$ & $4 q$ & $80 \%$ \\
\hline 18 & $2-\mathrm{Br}$ & $\mathrm{H}$ & $\mathrm{H}$ & $\mathrm{O}$ & $4 r$ & $76 \%$ \\
\hline 19 & $2,4-\mathrm{Cl}$ & $\mathrm{H}$ & $\mathrm{H}$ & $\mathrm{O}$ & $4 s$ & $82 \%$ \\
\hline 20 & $4-\mathrm{NO}_{2}$ & $\mathrm{Me}$ & $\mathrm{H}$ & $\mathrm{O}$ & $4 t$ & $84 \%$ \\
\hline 21 & $4-\mathrm{Cl}$ & $\mathrm{Me}$ & $\mathrm{H}$ & $\mathrm{O}$ & $4 u$ & $80 \%$ \\
\hline
\end{tabular}

${ }^{\mathrm{a}}$ Isolated yield ${ }^{\mathrm{b}} \mathrm{A}$ mixture of $o$-phenylenediamine or 2-aminophenol $(0.25$ $\mathrm{mmol})$, dimedone $(0.25 \mathrm{mmol})$ a benzaldehyde derivative $(0.25 \mathrm{mmol})$ and the DES (choline chloride/urea 1:2) were stirred vigorously at $80^{\circ} \mathrm{C}$.

Various benzaldehydes containing electron-donating, electronwithdrawing and halogen substituents were successfully applied to the reaction and afforded the benzo-fused seven-membered heterocycles in good to excellent yields. Electron-withdrawing groups on benzaldehyde showed increased yields in comparison to electron-donating groups.
Previous methods for the synthesis of 1,4-benzoxazepines involved performing the condensation reaction in dichloroethane, a toxic organic solvent, in the presence of trifluoroacetic acid with longer reaction times at high temperatures. ${ }^{37}$ However, in the presented methodology, the target 1,4-benzoxazepines were synthesized without acid catalysts, organic solvents or harsh reaction conditions. The results of the presented protocol were compared to that of recent reports. ${ }^{38-42}$ As shown in Table 2, the presented methodology has several advantages over the reported methodologies, such as a green chemical approach, straightforward and rapid procedure, without the need of any metals and a simple work-up protocol.

Table 2. Comparison of the results for the synthesis of 1,4benzodiazepine $\mathbf{4 b}$ using DES with reported methods.

\begin{tabular}{lllll}
\hline Entry & Conditions & $\begin{array}{l}\mathrm{T} \\
\left({ }^{\circ} \mathrm{C}\right)\end{array}$ & $\begin{array}{l}\text { Time } \\
(\mathrm{min})\end{array}$ & $\begin{array}{c}\text { Yield } \\
(\%)\end{array}$ \\
\hline 1 & $\mathrm{CF}_{3} \mathrm{COOH}-\mathrm{EtOH}$ & reflux & 45 & $70^{38}$ \\
2 & $\mathrm{ZnS} \mathrm{NPs-EtOH}$ & reflux & 10 & $85^{38}$ \\
3 & $\mathrm{ZnS} \mathrm{NPs-grinding}$ & 70 & 19 & $85^{39}$ \\
4 & $\mathrm{Fe}_{3} \mathrm{O}_{4} @$ chitosan-EtOH & r.t. & 120 & $91^{40}$ \\
5 & $\mathrm{Fe}(\mathrm{OTs})_{3} / \mathrm{SiO}_{2}$-S.F. & 80 & 5 & $96^{41}$ \\
6 & $\mathrm{Cu} / \mathrm{GA} / \mathrm{Fe}_{3} \mathrm{O}_{4} @ \mathrm{SiO}_{2} \mathrm{NPs}-$ & 60 & 40 & $95^{42}$ \\
& $\mathrm{EtOH}$ & & & \\
7 & DES & $\mathbf{8 0}$ & $\mathbf{2 0}$ & $\mathbf{9 2}$ \\
\hline
\end{tabular}

A possible mechanism for the formation of benzodiazepine derivatives is shown in Scheme 2. Firstly, the DES can activate the carbonyl group of dimedone $\mathbf{2}$ which then undergoes facile condensation with $\boldsymbol{o}$-phenylenediamine $\mathbf{1}$ to form an intermediate imine [A]. The $\mathrm{NH}_{2}$ group of $[\mathbf{A}]$ condenses with the DES activated carbonyl group of benzaldehyde derivatives $\mathbf{3}$ to form [B], which then undergoes an intramolecular cyclization to afford the products 4. Benzoxazepine derivatives may undergo an analogous mechanism in which intermediate $[\mathbf{B}]$ is replaced by the corresponding oxonium intermediate. DES can play a dual role in this reaction: (i) as a solvent and (ii) as an organocatalyst which activates the carbonyl and imine functional groups via hydrogen bonding.

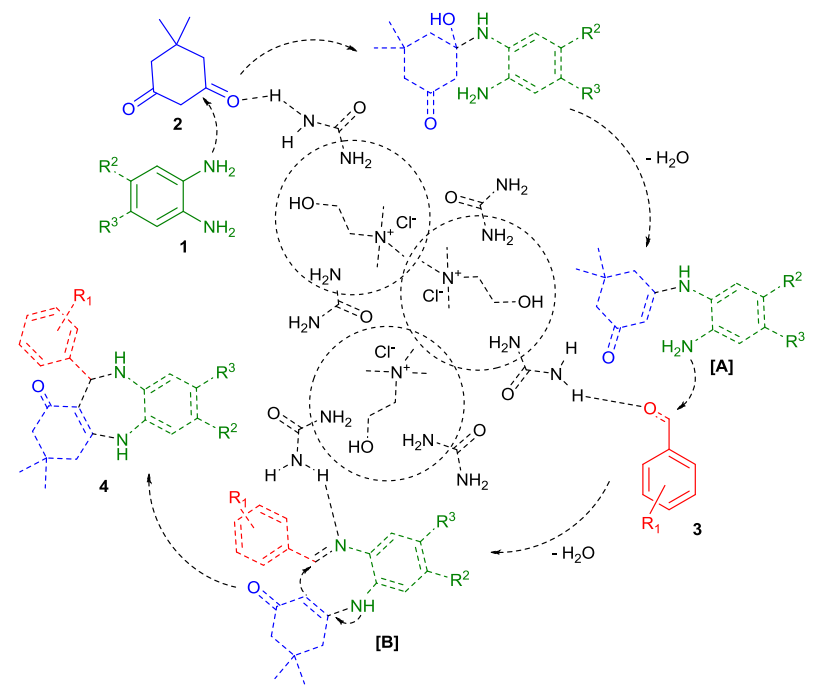

Scheme 2. Proposed pathway for the synthesis of benzo-fused sevenmembered heterocycles in choline chloride/urea 
Another advantage of using DESs is their ability to act as a recyclable reaction media. The reaction mixture, including the DES and $\mathbf{4 b}$, was dissolved in water and the insoluble crude product was filtered off. The DES was recovered from water by evaporation at $80{ }^{\circ} \mathrm{C}$ under vacuum, recycled and reused for four reaction runs without significant loss of catalytic activity (Fig. 2).

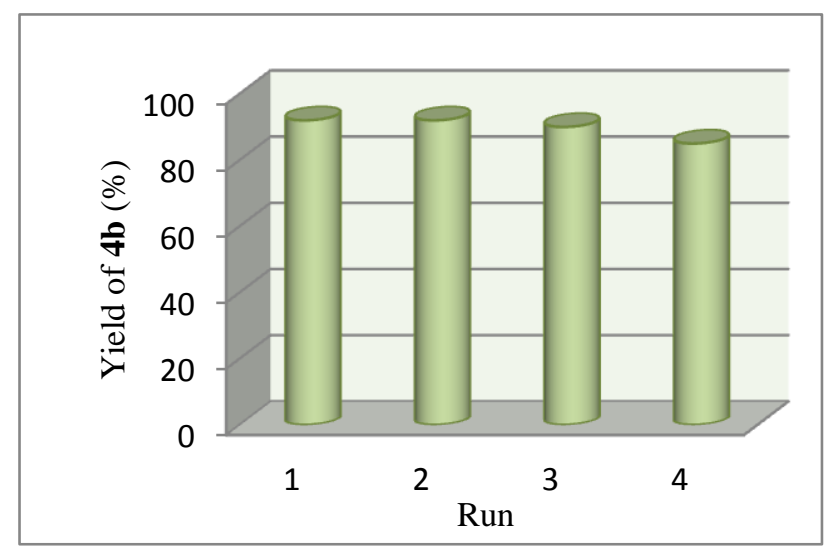

Figure 2. Reusability of DES for the synthesis of $\mathbf{4 b}$

\section{Conclusions}

In summary, we have developed a convenient and highly efficient method for the synthesis of benzo-fused sevenmembered heterocycles, including tricyclic 1,4-benzodiazepines and 1,4-benzoxazepines, using a DES as a solvent/organocatalyst. The mild reaction conditions, experimental simplicity, straightforward purification procedures, excellent yields with short reaction times, as well as the application of green chemistry principles, are the advantages of this methodology. This protocol may find use in synthetic and medicinal chemistry since the products contain structural and functional groups present in biologically active molecules.

\section{Acknowledgments}

We gratefully acknowledge financial support from the Iran National Science Foundation (INSF), the Research Council of Shahid Beheshti University and Catalyst Center of Excellence (CCE) at Shahid Beheshti University.

\section{References}

1 Wagle, D. V.; Zhao, H.; Baker, G. A. Acc. Chem. Res. 2014, 47, 2299.

2 Smith, E. L.; Abbott, A. P.; Ryder, K. S. Chem. Rev. 2014, 114, 11060.

3 Yusof, R.; Abdulmalek, E.; Sirat, K.; Rahman, M. B. A. Molecules 2014, 19, 8011.

4 Malaquias, J. C.; Steichen, M.; Thomassey, M.; Dale, P. J. Electrochim. Acta. 2013, 103, 15.

5 Liao, H. G.; Jiang, Y. X.; Zhou, Z. Y.; Chen, S. P.; Sun, S. G. Angew. Chem. 2008, 120, 9240.

6 Weaver, K. D.; Kim, H. J.; Sun, J.; MacFarlane, D. R.; Elliott, G. D. Green Chem. 2010, 12, 507.

7 Dai, Y.; van Spronsen, J.; Witkamp, G.-J.; Verpoorte, R.; Choi, Y. H. J. Nat. Prod. 2013, 76, 2162.

8 Arnold, T.; Jackson, A. J.; Sanchez-Fernandez, A.; Magnone, D.; Terry, A. E.; Edler, K. J. Langmuir. 2015, 31, 12894.

9 Liu, P.; Hao, J.-W.; Mo, L.-P.; Zhang, Z.-H. RSC Adv. 2015, 5, 48675.

10 Alonso, D. A.; Baeza, A.; Chinchilla, R.; Guillena, G.; Pastor, I. M.; Ramón, D. J. Eur. J. Org. Chem. 2016, DOI: 10.1002/ejoc.201501197.

11 Halimehjani, A. Z.; Namboothiri, I. N.; Hooshmand, S. E. RSC Adv. 2014, 4, 48022.

12 Halimehjani, A. Z.; Namboothiri, I. N.; Hooshmand, S. E. RSC Adv. 2014, 4, 51794

13 Estévez, V.; Villacampa, M.; Menéndez, J. C. Chem. Soc. Rev. 2014, $43,4633$.

14 Domling, A.; Wang, W.; Wang, K. Chem. Rev. 2012, 112, 3083.

15 Sternbach, L. H. The Benzodiazepine Story; Springer, 1978.

16 Bremner, J. B.; Samosorn, S. Azepines and their Fused-Ring Derivatives. 2008.

17 Cepanec, I.; Litvic, M.; Pogorelic, I. Org. Process Res. Dev. 2006, 10, 1192.

18 Miki, T.; Kori, M.; Mabuchi, H.; Tozawa, R.-i.; Nishimoto, T.; Sugiyama, Y.; Teshima, K.; Yukimasa, H. J. Med. Chem. 2002, 45, 4571.

19 Smits, R. A.; Lim, H. D.; Stegink, B.; Bakker, R. A.; de Esch, I. J.; Leurs, R. J. Med. Chem. 2006, 49, 4512.

20 Ursini, A.; Capelli, A. M.; Carr, R. A.; Cassarà, P.; Corsi, M.; Curcuruto, O.; Curotto, G.; Dal Cin, M.; Davalli, S.; Donati, D. J. Med. Chem. 2000, 43, 3596.

21 Bihel, F.; Kraus, J.-L. Org. Biomol. Chem. 2003, 1, 793.

22 Essaber, M.; Baouid, A.; Hasnaoui, A.; Benharref, A.; Lavergne, J.-P. Synth. Commun. 1998, 28, 4097.

23 Klunder, J. M.; Hargrave, K. D.; West, M.; Cullen, E.; Pal, K.; Behnke, M. L.; Kapadia, S. R.; McNeil, D. W.; Wu, J. C.; Chow, G. C. J. Med. Chem. 1992, 35, 1887.

24 Li, R.; Farmer, P. S.; Wang, J.; Boyd, R. J.; Cameron, T. S.; Quilliam, M. A.; Walter, J. A.; Howlett, S. E. Drug Des. Discov. 1995, 12, 337.

25 Nagarajan, K.; David, J.; Kulkarni, Y.; Hendi, S.; Shenoy, S.; Upadhyaya, P. Eur. J. Med. Chem. 1986, 21, 21.

26 Hajishaabanha, F.; Shaabani, A. RSC Adv. 2014, 4, 46844.

27 Shaabani, A.; Mofakham, H.; Maleki, A.; Hajishaabanha, F. J. Comb. Chem. 2010, 12, 630 .

28 Shaabani, A.; Rezayan, A. H.; Keshipour, S.; Sarvary, A.; Ng, S. W. Org. Lett. 2009, 11, 3342.

29 Shaabani, A.; Soleimani, E.; Rezayan, A. H.; Sarvary, A.; Khavasi, H. R. Org. Lett. 2008, 10, 2581.

30 Shaabani, A.; Ghadari, R.; Sarvary, A.; Rezayan, A. H. J. Org. Chem. 2009, 74, 4372.

31 Shaabani, A.; Sarvary, A.; Ghasemi, S.; Rezayan, A. H.; Ghadari, R.; Ng, S. W. Green Chem. 2011, 13, 582

32 Halimehjani, A. Z.; Hooshmand, S. E.; Shamiri, E. V. Tetrahedron Lett. 2014, 55, 5454 .

33 Shaabani, A.; Soleimani, E.; Maleki, A. Tetrahedron Lett. 2006, 47, 3031 .

34 Shaabani, A.; Hooshmand, S. E.; Tabatabaei, A. T. Tetrahedron Lett. 2016, 57,351 .

35 Shaabani, A.; Hooshmand, S. E. Tetrahedron Lett. 2016, 57, 310.

36 Shaabani, A.; Afshari, R.; Hooshmand, S. Res. Chem. Intermed. 2016, 42, 5607.

37 Ghandi, M.; Momeni, T.; Nazeri, M. T.; Zarezadeh, N.; Kubicki, M. Tetrahedron Lett. 2013, 54, 2983.

38 Naeimi, H.; Foroughi, H. New J. Chem. 2015, 39, 1228.

39 Naeimi, H.; Foroughi, H. Res. Chem. Intermed. 2016, 42, 3999.

40 Maleki, A.; Kamalzare, M. Tetrahedron Lett. 2014, 55, 6931.

41 Elwahy, A. H.; Shaaban, M. R. RSC Adv. 2015, 5, 75659.

42 Shaabani, A.; Sepahvand, H.; Hooshmand, S. E.; Borjian Boroujeni, M. Appl. Organomet. Chem. 2016, 30, 414. 
